\title{
PP40: Molecular diversity and population structure of the Ethiopian lentil (Lens culinaris Medikus) genotype assessment using SSR markers
}

Fikru Mekonnen ${ }^{1}$, Firew Mekbib ${ }^{2}$, Shiv Kumar ${ }^{3}$, Seid Ahmed ${ }^{3}$ and TR Sharma ${ }^{4}$

${ }^{1}$ Wello University, Ethiopia; ${ }^{2}$ College of Agriculture and Environmental Sciences, Haramaya University, Ethiopia; ${ }^{3}$ International Center for Agricultural Research in the Dry Areas (ICARDA), Rabat, Morocco; ${ }^{4}$ Chaudhary Sarwan Kumar Himachal Pradesh Agricultural University, Palampur, India. *(tiewoast@ gmail.com)

Knowledge of genetic diversity is essential for formulating effective germplasm collection, conservation and utilization strategies in crop improvement programs. It also provides an opportunity to take corrective steps infusing new genes to avoid risks associated with a narrow genetic base. Here, we analysed the genetic diversity of 119 lentil genotypes, including 83 germplasm accessions from Ethiopia and 36 exotic accessions from International Center for Agricultural Research in the Dry Areas, using 27 simple sequence repeat (SSR) markers. Analysis Molecular of Variance estimated the variations of $82 \%$ within and $18 \%$ among the populations. The degree of polymorphism observed among the populations was $100 \%$. A total of 122 alleles were detected, which ranged from 2 to 7 alleles per locus, with a mean of 4.52 alleles per locus. The estimated gene diversity for 27 loci was 0.64 . The average Shannon's information index value of 1.19, showed the existence of high genetic variation within the genotypes. The genetic similarity indices ranged from 0.21 to 1.00 . The SSR markers showed an average polymorphic information content (PIC) value of 0.58 . Cluster analysis grouped the genotypes into five major clusters as distinct genetic populations. This molecular diversity information provides a basis for future germplasm collection, utilization, and conservation strategies in gene banks and introducing exotic germplasm to widen the genetic base of the current lentil breeding populations. 